\title{
Searching the INTEGRAL WEAK alert population for Gamma-ray Bursts and other high energy transients
}

\section{A. B. Higgins*}

Department of Physics and Astronomy, University of Leicester, University Road, Leicester LE1 $7 R H, U K$.

E-mail: abh13ele.ac.uk

\section{R. L. C. Starling}

Department of Physics and Astronomy and Leicester Institute for Space and Earth Observation, University of Leicester, University Road, Leicester LE1 7RH, UK.

E-mail: rlcslele.ac.uk

\section{Götz}

AIM-CEA/DRF/Irfu/Service d'Astrophysique, Orme des Merisiers, 91191 Gif-sur-Yvette, France. E-mail: diego.gotz@cea.fr

\section{S. Mereghetti}

INAF, IASF-Milano, via E. Bassini 15, 20133 Milano, Italy.

E-mail: sandrodiasf-milano.inaf.it

\begin{abstract}
We investigate the nature of WEAK alerts, when the instruments detect an event that falls below the regular significance thresholds. We have initiated rapid follow-up observations of a sample of these alerts using the Swift satellite to allow for a broadband analysis of the alerts. Out of 15 chosen WEAK alert triggers $(\approx 4 \%$ of the entire WEAK trigger population) we confirm the existence of six GRBs and, additionally, one candidate AGN. We have shown that real high energy events exist within the INTEGRAL WEAK alert population. Future investigations into WEAK alerts could further explore the low fluence regime for explosive transients and give direction for future gamma-ray missions.
\end{abstract}

11th INTEGRAL Conference Gamma-Ray Astrophysics in Multi-Wavelength Perspective, 10-14 October 2016

Amsterdam, The Netherlands

* Speaker. 


\section{Introduction}

Gamma-ray Bursts (GRBs) are some of the most energetic electromagnetic events in the universe (Gehrels \& Mészáros 2012). Classically they are divided into two sub-groups; long and short duration GRBs (Kouveliotou et al. 1993). Long duration GRBs are thought to be produced due to the collapse of massive stars (Woosley \& Bloom 2006) and short duration due to the coalescence of compact binary mergers (Berger 2014). GRBs span a very large range of luminosities, typically $10^{45} \leq \mathrm{L}_{\mathrm{ISO}} \leq 10^{54} \mathrm{erg} \mathrm{s}^{-1}$. Many previous investigations into GRB luminosity functions have implied the existence of a population of low-luminosity GRBs (Daigne et al. 2007; Liang et al. 2007) with further investigations concluding that a number of these GRBs could exist as a local population (Norris 2002; Norris et al. 2005; Chapman et al. 2007).

We look at two currently active gamma-ray missions - The INTErnational Gamma-Ray Astrophysics Laboratory (INTEGRAL; Winkler et al. 2003) and Swift (Gehrels et al. 2004). INTEGRAL is a general purpose gamma-ray observatory but has also contributed to GRB science thanks to the INTEGRAL Burst Alert System ${ }^{1}$ (IBAS; Mereghetti et al. 2003) which has localized 119 GRBs (correct as of 1 June 2017). The INTEGRAL IBIS instrument (Ubertini et al. 2003) is more sensitive than the Swift Burst Alert Telescope (BAT; Barthelmy et al. 2004) at energies of $\approx 15-200$ $\mathrm{keV}$, with the capability of reaching lower peak flux limits $>50 \mathrm{keV}$ (Bosnjak et al. 2014). Additionally, an investigation by Foley et al. (2008) suggested that INTEGRAL may detect a local, low-luminosity GRB population. We chose a selection of INTEGRAL IBAS WEAK alerts for further analysis to test whether INTEGRAL can detect real high energy transients at these sensitivities. In addition to the BAT, Swift has the X-Ray Telescope (XRT; Burrows et al. 2005) and the Ultraviolet/Optical Telescope (UVOT; Roming et al. 2005). Swift is also capable of slewing towards a BAT detected or manually selected target. This allows us to complement the INTEGRAL data with broadband follow-up observations to fully characterise the properties of the alerts.

\section{IBAS alerts and our selection of WEAK alerts}

The IBAS software running at the INTEGRAL Science Data Centre (ISDC; Courvoisier et al. 2003) makes it possible to detect and localize GRBs in real time. IBAS has two significance threshold levels, named STRONG and WEAK which determine the distribution of the alerts. Prior to 26 January 2011 STRONG alerts were publicly distributed but alerts below this threshold, the WEAKs, were only distributed among the IBAS team. Since then all WEAK alerts have been public making rapid follow-up analysis possible.

There have been 402 WEAK alert triggers (number correct as of 1 July $1^{\text {st }} 2016$ ) of which we chose 15 to follow up with Swift (see table 1). The triggers comprised of the following:

- 11 chosen triggers that had not been detected by the Swift BAT. We requested target of opportunity (ToO) observations for multi-wavelength follow-up.

- Two WEAK triggers where ToOs had previously been requested elsewhere and had XRT data available for analysis.

\footnotetext{
${ }^{1}$ http://ibas.iasf-milano.inaf.it/
} 
- Two WEAK triggers that simultaneously triggered the Swift BAT and had XRT data available for analysis.

The 11 'chosen triggers' we selected for further follow-up were chosen based on a set of criteria where each trigger had to meet at least one:

- Trigger significance close to the $8 \sigma$ STRONG threshold to increase the probability of the trigger being a result of a real event.

- Trigger positions were cross-matched with catalogues to rule out nearby or coincident existing X-ray sources and high Galactic extinction.

- Triggers were followed up with a ToO request if the trigger time coincided with the working hours of the Swift team on-call member.

\begin{tabular}{|c|c|c|c|c|c|}
\hline $\begin{array}{c}\text { ToO } \\
\text { Name }\end{array}$ & $\begin{array}{c}\text { IBAS } \\
\text { Trigger No. }\end{array}$ & $\begin{array}{c}\text { IBAS trigger } \\
\text { significance } \\
(\sigma)\end{array}$ & $\begin{array}{c}\text { RA } \\
(\mathrm{Deg}) \\
(\mathrm{J} 2000)\end{array}$ & $\begin{array}{c}\text { Dec } \\
(\mathrm{Deg}) \\
(\mathrm{J} 2000)\end{array}$ & $\begin{array}{c}\text { Localization } \\
\text { Error } \\
(\mathrm{arcmin})\end{array}$ \\
\hline IGRW 160610 & $7488 / 0$ & 6.7 & 359.90 & 61.57 & 3.8 \\
\hline IGRW 151019 & $7277 / 0$ & 7.0 & 292.82 & 31.14 & 3.5 \\
\hline IGRW 150903 & $7231 / 0$ & 6.7 & 239.17 & -33.81 & 3.6 \\
\hline IGRW 150831* & $7228 / 0$ & 7.3 & 220.98 & -25.65 & 3.4 \\
\hline IGRW 150610 & $7005 / 0$ & 7.1 & 178.32 & 16.03 & 4.8 \\
\hline IGRW 150305 & $6905 / 0$ & 7.6 & 269.79 & -42.62 & 3.4 \\
\hline IGRW 140219 & $6467 / 0$ & 6.7 & 204.10 & -45.06 & 3.6 \\
\hline IGRW 130904 & $6931 / 0$ & 6.7 & 256.88 & -32.01 & 3.6 \\
\hline IGRW 121212* & $6720 / 0$ & 7.9 & 177.90 & 78.00 & 3.3 \\
\hline IGRW 110718 & $6323 / 0$ & 6.8 & 256.78 & 40.05 & 3.6 \\
\hline IGRW 110608 & $6297 / 0$ & 6.8 & 315.28 & 32.041 & 3.6 \\
\hline IGRW 110428 & $6169 / 0$ & 7.2 & 320.27 & -33.96 & 3.5 \\
\hline IGRW 110112 & $6127 / 0$ & 7.4 & 10.56 & 64.41 & 2.6 \\
\hline IGRW 100909* & $6060 / 0$ & 7.7 & 73.95 & 54.65 & 2.0 \\
\hline IGRW 091111* & - & 7.2 & 137.81 & -45.91 & 2.9 \\
\hline
\end{tabular}

Table 1: Table containing the IBAS trigger properties of the 15 WEAK triggers. The naming convention for the WEAK triggers is IGRW YYMMDD prior to our identification of any potential source. The Trigger No., alert significance $(\sigma)$, RA, Dec and localization error (90 per cent confidence) were all taken from IBAS.

${ }^{*}$ The four triggers we did not choose directly but had existing XRT data available.

This investigation into the INTEGRAL WEAK alert population analyses $\approx 4 \%$ of the total WEAK triggers. We do not aim to characterise the entire sample and cannot claim our selection process of triggers gives us a complete sample. We aim to produce a study probing whether real high energy transients exist within the population. 


\section{Swift broadband follow-up analysis}

The Swift data used during this investigation were made available by the UK Swift Science Data Centre (UKSSDC; Evans et al. 2007, 2009). We analysed the ToO XRT and UVOT data for the 15 WEAK triggers to determine the broadband nature of the alerts. We used the Swift XRT pipeline tool to produce cleaned event files which were searched for any real sources (with $>3 \sigma$ detection significance) within the IBAS error region. If a source was detected further ToOs were requested to determine if the X-ray source was fading. Upper-limits were calculated for nondetections. For the four triggers with existing XRT data we used the XRT products made available by the UKSSDC. All positive detections were cross-referenced with the Vizier source catalogue database (Ochsenbein et al. 2000) to check for any existing X-ray sources coincident with the detection position. Out of our 15 WEAK alerts we obtained positive detections for six triggers (see figure 1). IGRW 110112 was not detected with the XRT but later confirmed as a GRB via a simultaneous observation by Fermi GBM (see Connaughton 2011).

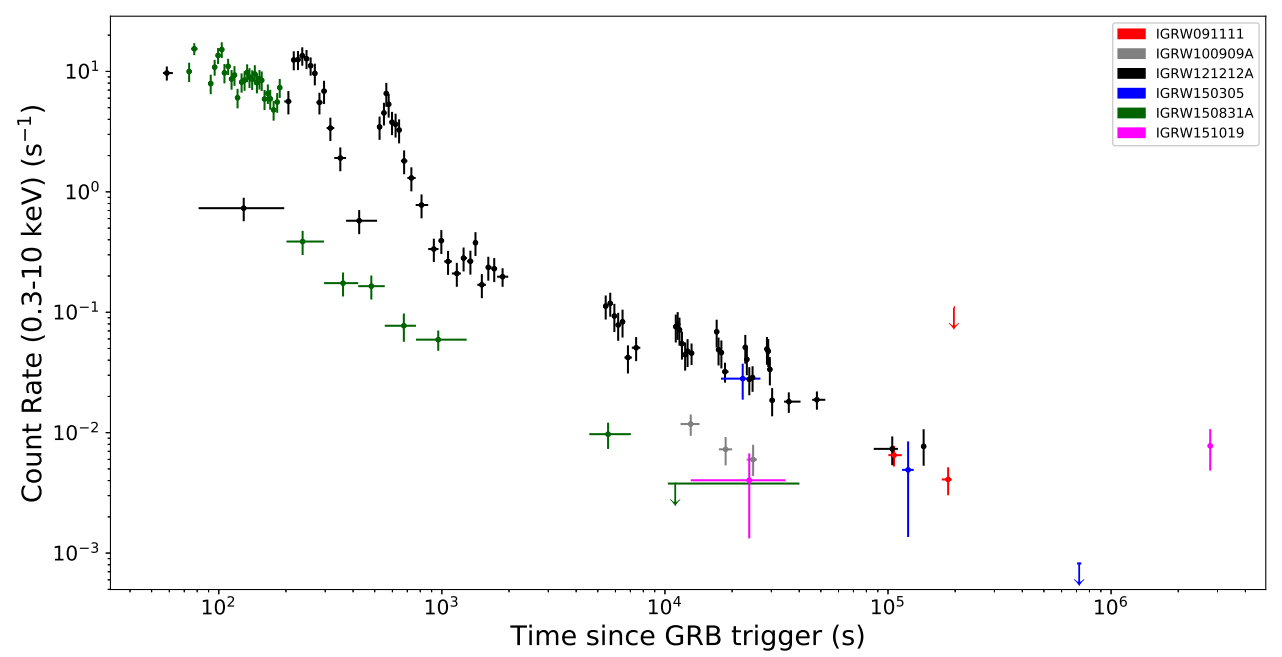

Figure 1: X-ray count rates/afterglows of the six WEAK triggers detected by the Swift XRT.

Five of the six detections were confirmed to have fading x-ray sources, behaviour typical of a GRB (O'Brien et al. 2006). This included IGRW 150305/GRB 150305A a new GRB classified during this investigation that had not triggered or been observed by other gamma-ray observatories (see figure 2). IGRW 151019 on the other hand showed no signs of fading after $\approx 1$ month and spectral analysis performed fitting an absorbed power law revealed a photon index of $\Gamma \approx 1.7 \pm 0.4$. These properties are similar to those found for AGN (Nandra \& Pounds 1994; Brightman \& Nandra 2011). When cross-referenced with Vizier, all XRT detections were found to have no existing catalogued X-ray sources.

We analysed the UVOT data for each positive X-ray detection to determine if any coincident sources could be found at UV/Optical wavelengths. The UVOT data comprised of several images per exposure for each UVOT filter. We summed all images over all exposures to create one image covering the entire observation of the source for each filter. To search for a coincident source we 

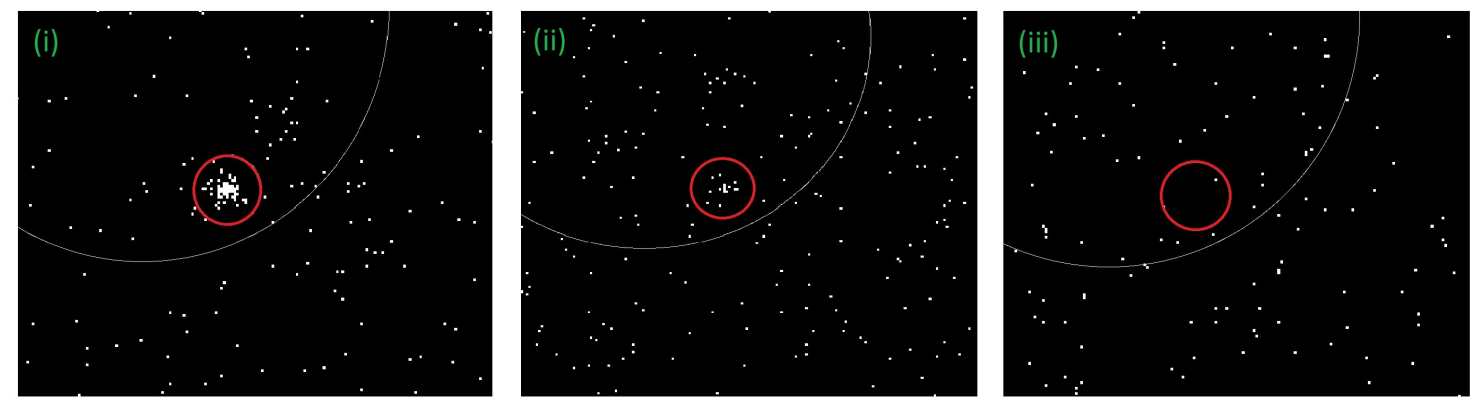

Figure 2: Images showing a significant detection of an X-ray source (red) within the INTEGRAL error region (yellow) of IGRW/GRB 150305 which clearly fades over time. Observations correspond to $17000-27000$ s (i), $120000-130000 \mathrm{~s}$ (ii) and $700000-740000 \mathrm{~s}$ (iii) after the GRB occurred.

used the Swift UVOTSOURCE tool. We set a significance of $3 \sigma$ to distinguish between a detection and an upper limit (see Breeveld et al. 2010). An optical source was detected coincident with the position of IGRW 121212/GRB 121212A (white filter) lying at RA, Dec (J2000) 177.79341, 78.03780 deg with a 0.48 arcsec uncertainty $(1 \sigma)$. Vizier reported no previously catalogued optical source. Additionally, a UV source was detected coincident with the position of IGRW 151019 at RA, Dec (J2000) 292.78334, 31.13252 deg with a 0.49 arcsec uncertainty $(1 \sigma)$. The AllWISE source J193108.05+310756.4 (Cutri et al. 2014) was coincident to the UVOT source and lay $\approx 1.8$ arcsec from the XRT centroid. IGRW100909/GRB 100909A was not detected with the UVOT but an optical afterglow was confirmed via observations from both the Faulks Telescope North and Nordic Optical Telescope (Guidorzi et al. 2010; Jelinek et al. 2010). See Higgins et al. (2017) for full details on the Swift follow-up analysis.

\section{Discussion and conclusions}

We show that real high energy events exist within the INTEGRAL IBAS WEAK alert population, classifying six GRBs and a candidate AGN out of 15 chosen triggers. One of the discovered GRBs, GRB 150305, was not triggered or observed with any other gamma-ray observatories and only classified as a result of this investigation. We highlight that we have only analysed $\approx 4 \%$ of the total population and cannot make general conclusions for the number of real events within the entire sample. However, to date 17 of the $119(\approx 14 \%)$ GRBs localised by IBAS were sub-threshold alerts suggesting that reducing the trigger thresholds for automated analysis for current and future missions would lead to the detection and discovery of additional transient events such as GRBs.

\section{References}

[1] N. Gehrels, P. Mészáros, Gamma-ray Bursts, Science, 337, 932, (2012)

[2] C. Kouvelioutou, C. Meegan, A. Fishman, G. J. Bhat, N. P. Briggs, T. M. Koshut, Identification of two classes of gamma-ray bursts, ApJ, 413, L101 (1993)

[3] S. E. Woosley, \& J. S. Bloom, The Progenitor Stars of Gamma-Ray Bursts, Ann. Rev. Astron. Astrophys., 44, 507 (2006) 
[4] E. Berger, Short-duration gamma-ray bursts, Ann. Rev. Astron. Astrophys., 52, 43 (2014)

[5] F. Daigne, R. Mochkovitch, The low-luminosity tail of the GRB distribution: the case of GRB 980425 A\&A, 465, 1, (2007)

[6] E. Liang, B. Zhang, F. Virgili, Z. G. Dai, Low-Luminosity Gamma-Ray Bursts as a Unique Population: Luminosity Function, Local Rate, and Beaming Factor, ApJ, 662, 1111 (2007)

[7] J. P. Norris, Implications of the Lag-Luminosity Relationship for Unified Gamma-Ray Burst Paradigms, ApJ, 579, 386 (2002)

[8] J. P. Norris, J. T. Bonnell, D. Kazanas, J. D. Scargle, J. Hakkila, T. W. Giblin, Long-Lag, Wide-Pulse Gamma-Ray Bursts, ApJ, 627, 324 (2005)

[9] R. Chapman, N. R. Tanvir, R. S. Priddey, A. J. Levan, How common are long gamma-ray bursts in the local Universe? MNRAS, 382, L21 (2007)

[10] C. Winkler, et al., The INTEGRAL mission, A\&A, 411, L1 (2003)

[11] N. Gehrels, et al., The Swift Gamma-Ray Burst Mission, ApJ, 611, 1005 (2004)

[12] S. Mereghetti, D. Götz, J. Borkowski, R. Walter, H. Pedersen, The INTEGRAL Burst Alert System, A\&A, 411, L291 (2003)

[13] P. Ubertini, et al., IBIS: The Imager on-board INTEGRAL, A\&A, 411, L131 (2003)

[14] S. D. Barthelmy, The Burst Alert Telescope (BAT) on the Swift MIDEX mission, Society of Photo-Optical Instrumentation Engineers (SPIE) Conference Series, 5165, X-Ray and Gamma-Ray Instrumentation for Astronomy XIII, 175, (2004)

[15] Ž. Bošnjak, D. Götz, L. Bouchet, S. Schanne, B. Cordier, The spectral catalogue of INTEGRAL gamma-ray bursts. results of the joint IBIS/SPI spectral analysis, A\&A, 561, A25 (2014)

[16] S. Foley, S. McGlynn, L. Hanlon, S. McBreen, B. McBreen, Global characteristics of GRBs observed with INTEGRAL and the inferred large population of low-luminosity GRBs, A\&A, 484, 143 (2008)

[17] D. N. Burrows, et al., The Swift X-Ray Telescope, Space Sci. Rev., 120, 165 (2005)

[18] P. W. A. Roming, et al., The Swift Ultra-Violet/Optical Telescope, Space Sci. Rev., 120, 95 (2005)

[19] T. J.-L. Courvoisier, et al., The INTEGRAL Science Data Centre (ISDC), A\&A, 411, L53 (2003)

[20] P. A. Evans, et al., An online repository of Swift/XRT light curves of $\gamma$-ray bursts, A\&A, 469, 379 (2007)

[21] P. A. Evans, et al., Methods and results of an automatic analysis of a complete sample of Swift-XRT observations of GRBs, MNRAS, 397, 1177 (2009)

[22] F. Ochsenbein, P. Bauer, J. Marcout, The VizieR database of astronomical catalogues, A\&AS, 143, 23 (2000)

[23] V. Connaughton, GRB 110112B: Fermi GBM observation, GRB Coordinates Network, 11569 (2011)

[24] P. T. O'Brien, et al., The early X-ray emission from GRBs, ApJ, 647, 1213 (2006)

[25] K. Nandra, K. A. Pounds, GINGA Observations of the X-Ray Spectra of Seyfert Galaxies, MNRAS, 268, 405 (1994)

[26] M. Brightman, K. Nandra, An XMM-Newton spectral survey of $12 \mu \mathrm{m}$ selected galaxies - I. X-ray data, MNRAS, 413, 1206 (2011) 
[27] A. A. Breeveld, et al., Further calibration of the Swift ultraviolet/optical telescope, MNRAS, 406, 1687 (2010)

[28] R. M. Cutri, et al. VizieR Online Data Catalog: AllWISE Data Release, 2328 (2014)

[29] C. Guidorzi, Z. Cano, D. Bersier, P. O’Brien, GRB 100909A: Faulkes Telescope North afterglow candidate, GRB Coordinates Network, 11265 (2010)

[30] M. Jelinek, J. Gorosabel and S. Simon, GRB100909A: NOT optical afterglow confirmation, GRB Coordinates Network, 11269 (2010)

[31] A. B. Higgins, et al., Investigating the nature of INTEGRAL Gamma-ray Bursts and sub-threshold triggers with Swift follow-up, MNRAS, 470, 314 (2017) 